\title{
An unusual case of pneumatic nail gun scrotal injury and revision of the literature
}

\author{
Filippo Migliorini ${ }^{1}$, Leonardo Bizzotto ${ }^{1}$, Pierpaolo Curti ${ }^{2}$, Antonio Benito Porcaro ${ }^{1}$, Walter Artibani ${ }^{1}$ \\ ${ }^{1}$ Department of Urology, Azienda Ospedaliera Universitaria Integrata, Verona, Italy; \\ ${ }^{2}$ Department of Urology, Ospedale "Mater Salutis", Legnago, Verona, Italy.
}

\begin{abstract}
Summary Pneumatic nail guns are hand-held tools commonly utilized in both industrial and non occupational setting. These devices facilitate production and boost efficiency but also can be a potential cause of serious injuries. Nail guns are the most frequent tool associated trauma with hospitalization among construction workers. The most common sites of injuries are the hand or fingers followed by the lower extremities. We report the first case in literature of a work nail gun injury to male external genitalia.
\end{abstract}

KEY WORDS: Pneumatic nail gun; Injury; Scrotum; Epididymis.

Submitted 24 August 2017; Accepted 21 September 2017

\section{INTRODUCTION}

Pneumatic nailers increase production rates in many jobs and reduce manufactoring cost. These tools impart a large amount of energy to a small "projectile" that, if not properly aimed, it can be a potential cause of injury ranging from a slight scratch to serious life threatening trauma and death (1-2). Numerous cases of nail gun injuries have been documented involving not only the extremities but also brain, eyes, neck, heart, thorax, spinal cord, bowel and liver (3). According to our best knowledge, an external male genitalia pneumatic nail gun penetrating injury has never been described before.

\section{Case report}

A 53-year-old right handed Caucasian male, furniture maker presented to the local Emergency Department for a work pneumatic nail gun injury. The patient, while using a pneumatic nail gun equiped with Contact Actuation Trigger (CAT) security system, accidentally slipped and involuntarily fired a nail with sudden onset of scrotal pain. Physical examination pointed out a puncture wound in the left side of the scrotum, tenderness of the left testicle and epididymis with no signs of bleeding or haematoma. The X-ray rivealed a thin nail of about 20 $\mathrm{mm}$ in length in the left side of the scrotum (Figure la). A scrotal color Doppler ultrasound showed regular testicles and the presence of a thin linear hyperechoic foreign body at the head of left epididymis with no signs of haematoma. Blood tests were regular.

The patient, after been given prophylaxis with human tetanus immunoglobulin and antibiotic, underwent urgent surgical scrotal exploration. Almost completely inside the head of the left epididymis was detected a nail (Figure 1b) which was easily gently removed in a retrograde fashion. The patient was discharged the next day with no sequelae.

\section{Discussion}

Since 1959, when pneumatically powered nail guns were introduced in the construction industry, there has been an increasing number of accidents involving these devices (3-5). These tools are efficient, readily available, and easy to use, making them common employed in residential construction, wood-production industries and in non occupational setting (6).

Pneumatic nail guns have a safety device at the end of the gun muzzle that must be depressed before the fastener can be discharged. There are generally two types of trigger systems which then define how the nail gun fires in response to a trigger press: the Sequential Actuation Trigger (SAT) requires that each nail can only be discharged when the safety tip is first depressed and, while held depressed, the trigger is squeezed. The other one is the CAT which allows the operator to first squeeze the trigger and, while holding the trigger squeezed, repeatedly bump the safety tip on the work piece to shoot multiple nails. Traumatic injuries can occur when an operator intentionally discharges a nail using both types of actuation systems. However, a nail gun equipped with a SAT system is much less likely to be discharged uninten-

\section{Figure 1.}

a) the nail at X-ray;

b) the nail at the head of the left epididymis.

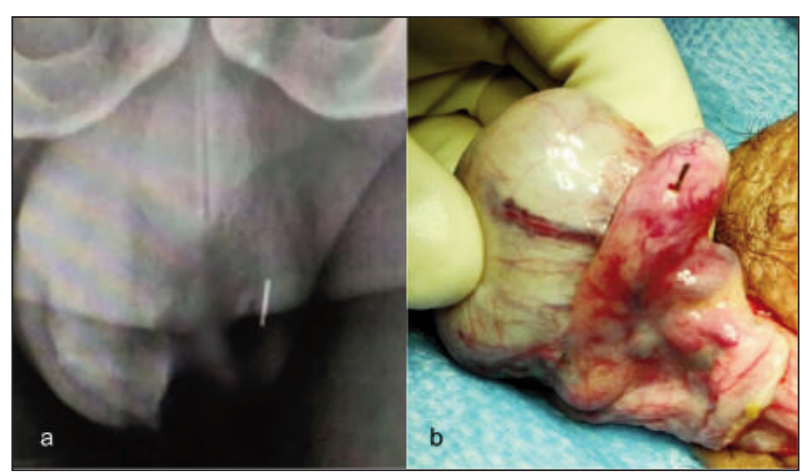


tionally, as the trigger must be activated while the safety tip is depressed against the body. Unintentional nail discharge using the CAT system typically takes place following nail gun recoil (resulting in a "double fire" - second unintended shot) or when the operator has their finger on the trigger and the nail gun nose inadvertently contacts an object $(1,7)$.

Gun nailers impart a large amount of energy to a small "projectile" witch can reach the velocity of up to 1500 $\mathrm{km} / \mathrm{h}$. The amount of energy required to cause serious injury is fairly low: penetrating of the skin occurs with "projectile" velocities of $165 \mathrm{~km} / \mathrm{h}$, whereas bony fractures may occur with a velocities of $215 \mathrm{~km} / \mathrm{h}$. When a nail penetrates human tissues the kinetic energy transfers from the object to the surrounding tissues. As the shock wave expand, the temporary cavity created causes crush and stretch damage to tissues $(8,9)$.

From 2006 to 2011 in US hospital EDs, a total of 151,000 injuries due to nail guns were treated and just over half of these were work-related. Puncture wounds and foreign bodies accounted for over $85 \%$ injuries, followed by fractures. Approximately $90 \%$ of the patients were treated and released and 10\% were treated and admitted. Injuries most commonly involved the hand/fingers in $56 \%$ of the cases, followed by the wrist or lower arm $(8.3 \%)$, foot or toes $(6.9 \%)$, knee $(4.8 \%)$, and eye (4.3\%) (10). Case reports have described injuries to the thorax, heart, abdominal wall, flank, pelvic wall, facial bones, skull. Paralytic spinal cord transection, bowel perforation, long bone fracture, liver laceration, hemopneumothorax, blindness, cerebral damage, and even fatal injuries have been reported (11-13). These trauma can also often be complicated by contamination with oil, paper, or glue and the combination of tissue edema, devitalized tissue, and foreign matter provides an ideal environment for local infection (14).

The evaluation of patient with a suspect of retained nail begins with a careful history and physical examination followed by an X Ray and, in some cases, an Ultrasound or Computed Tomography scan. Special attention should be given to the type of nail gun used and the mechanism of injury. Status of tetanus immunization must also be determined and antibiotic prophylaxis has to be given. In case of nail embedded or close to vascular/nervous structures a surgical exploration is mandatory (3).

Our unusual case of work scrotal injury due to a pneu- matic nail gun is the first reported in literature and required a surgical treatment. Since these tools will likely continue to be used, management can reduce worker exposure to nail-gun hazards by using a combination of engineering controls, administrative controls and personal protective equipment (7).

\section{REFERENCES}

1. Lipscomb HJ, Dement JM, Nolan J, et al. Nail gun injuries in residential carpentry: lessons from active injury surveillance. Inj Prev. 2003; 9:20-24.

2. Schaller BJ, Kleindienst A, Kruschat $T$, et al. Industrial nail gun injury to the anterior skull base: a case report and review of the literature. J Trauma. 2008; 64:E29-32.

3. Pierpont YN, Pappas-Politis E, Naidu DK, et al. Nail-gun injuries to the hand. Eplasty. 2008; 8:479-488.

4. Edlich RF, Silloway KA, Rodeheaver GT, et al. Industrial nail gun injuries. Compr Ther. 1986; 12:42-46.

5. Absoud EM, Harrop SN. Hand injuries at work. J Hand Surg Br. 1984; 9:211-5.

6. Hoffman DR, Jebson PJ, Steyers CM. Nail gun injuries of the hand. Am Fam Physician. 1997; 56:1643-6.

7. Albers J, Lowe B, Lipscomb H, et al. Revisiting pneumatic nail gun trigger recommendations. Prof Saf. 2015; 60:30-33.

8. Ordog GJ, Wasserberger J, Balasubramaniam S. Shotgun wound ballistics. J Trauma. 1988; 28:624-31.

9. Childs SA. Nail gun injury. Orthop Nurs. 1991; 10:15-8.

10. Lipscomb HJ, Schoenfisch AL. Nail gun injuries treated in U.S emergency departments, 2006-2011: not just a worker safety issue. Am J Ind Med. 2015; 58:880-885

11. Webb DP, Ramsey JJ, Dignan RJ, Drinkwater DC Jr. Penetrating injury to the heart requiring cardiopulmonary bypass: a case study. J Extra Corpor Technol. 2001; 33:249-51.

12. Jithoo R, Govender ST, Nathoo N. Penetrating nail gun injury of the head and chest with incidental pericallosal artery aneurysm. S Afr Med J. 2001; 91:316-7.

13. Wang MJ, Chen IS, Tsai SK. Nail gun penetrating injury of the left ventricle and descending aorta. Circulation. 1999; 100:e18-9.

14. Tikka S. The contamination of missile wounds with special reference to early antimicrobial therapy. Acta Chir Scand Suppl. 1982; 508:281-7.

\section{Correspondence}

Filippo Migliorini, MD (Corresponding Author)

filippo.migliorini@aovr.veneto.it

Leonardo Bizzotto, MD

Antonio Benito Porcaro, MD

Walter Artibani, MD

Department of Urology, Azienda Ospedaliera Universitaria Integrata

Piazzale Stefani 1, IT- 37126 Verona, Italy 